\title{
Aprendizaje Basado en Problemas una estrategia de enseñanza en la asignatura de Ciencias Naturales
}

\section{Problem-Based Learning a teaching strategy in the subject of Natural Sciences}

\author{
Luis Pillaga Guamán \\ Ipillagag@ucacue.edu.ec \\ Universidad Católica de Cuenca, Cañar \\ Ecuador \\ https://orcid.org/0000-0002-7660-7947 \\ Darwin Gabriel Garcia-Herrera \\ dggarciah@ucacue.edu.ec \\ Universidad Católica de Cuenca, Azogues \\ Ecuador \\ https://orcid.org/0000-0001-6813-8100 \\ Nancy Marcela Cárdenas-Cordero \\ ncardenasc@ucacue.edu.ec \\ Universidad Católica de Cuenca, Azogues \\ Ecuador \\ https://orcid.org/0000-0002-6250-6504 \\ Juan Carlos Erazo-Álvarez \\ jcerazo@ucacue.edu.ec \\ Universidad Católica de Cuenca, Cuenca \\ Ecuador \\ https://orcid.org/0000-0001-6480-2270
}

Recibido: 25 de abril de 2020

Revisado: 20 de mayo de 2020

Aprobado: 10 de junio de 2020

Publicado: 29 de junio de 2020 


\title{
RESUMEN
}

El objetivo de la investigación fue describir la participación de los educandos, a través de la aplicación del ABP como estrategia didáctica para la adquisición de conocimientos significativos en la asignatura de Ciencias Naturales. Se desarrolló desde una perspectiva cuantitativa, mediante el método hipotético deductivo y un tipo descriptiva correlacional transversal no experimental, mediante el cálculo de Chi-cuadrado de Pearson, con la finalidad de conocer la asociación de las variables de estudio. La estrategia didáctica del aprendizaje basado en problemas (ABP), impactó en los estudiantes a quienes les permitió el desarrollo de habilidades comunicativas, de trabajo en equipo e interpersonales; de competencias básicas y científicas; de una actitud positiva frente al aprendizaje y adicionalmente.

Descriptores: Educación a distancia; tecnología educacional; aprendizaje activo; epidemiología. (Palabras tomadas del Tesauro UNESCO).

\begin{abstract}
The objective of the research was to describe the participation of the students, through the application of the ABP as a didactic strategy for the acquisition of significant knowledge in the subject of Natural Sciences. It was developed from a quantitative perspective, using the deductive hypothetical method and a non-experimental crosssectional correlational descriptive type, using Pearson's Chi-square calculation, in order to know the association of the study variables. The didactic strategy of problem-based learning (ABP) impacted the students, who allowed them to develop communication, teamwork and interpersonal skills; basic and scientific skills; of a positive attitude towards learning and additionally.
\end{abstract}

Descriptors: Distance education; educational technology; activity learning; epidemiology. (Words taken from the UNESCO Thesaurus). 


\section{INTRODUCCIÓN}

El presente artículo de investigación tiene como propósito contribuir a mejorar la participación en los estudiantes dentro y fuera del aula clase, a través de dos enfoques, el Aprendizaje Basado en Problemas (ABP) como estrategia didáctica y la adquisición de conocimientos para un Mejoramiento Continuo, que permita fomentar habilidades de pensamiento crítico, aprendizaje significativo, en la cuarta unidad del texto de Santillana para octavo año de EGB del Colegio de Bachillerato Particular Justiniano Crespo Verdugo; según (Instituto Tecnológico y de Estudios Superiores de Monterrey, 2016) el ABP es importante porque enseña al estudiante los contenidos de la asignatura basándose en caso similares a los que el estudiante vive.

Ese realismo le ayuda a elaborar la información, alejándole del aprendizaje teórico, sin referencia a la realidad. Por consiguiente, la importancia radica en que como docentes se trabaja con el estudiante desde su contexto vivencial y en su ambiente adolescente para la enseñanza aprendizaje de las Ciencias Naturales, proyectándose la generación de proyectos en apoyo de las TIC, lo cual permite contextualizar a la nueva realidad social vivida a causa del COVID-19 (Vélez-Loor, et al., 2020).

Hoy en día el Aprendizaje Basada en Problemas, tiene características importantes como las que menciona (Campos, 2013), el tema de estudio por el estado actual de la educación en emprendimiento a partir del análisis de las principales tendencias teóricas e investigaciones relacionadas con el tema, así como documentos de políticas y la investigación sobre el emprendimiento en la enseñanza y el aprendizaje; por lo que la este tipo de estrategia se convierte en una ayuda para la enseñanza de las Ciencias Naturales:

La enseñanza de las Ciencias Naturales favorece a los niños y jóvenes en el desarrollo de sus capacidades de observación, análisis, razonamiento, comunicación, por ende, la tarea del docente debe estar orientada hacia el desarrollo de su pensamiento crítico y reflexivo de manera autónoma, para lo cual debe basarse en diferentes estrategias de 
enseñanza y aprendizaje como ABP, recursos que ayudan al maestro a cumplir con sus objetivos.

Con todos estos antecedentes el objetivo de la investigación fue describir la participación de los educandos, a través de la aplicación del ABP como estrategia didáctica para la adquisición de conocimientos significativos en la asignatura de Ciencias Naturales.

\section{Referencial teórico}

Es importante dentro del documento investigativo desarrollar los diferentes temas que permitan mejorar y fundamentar el tema de interés relacionado a la enseñanza de las Ciencias Naturales por medio de la aplicación de estrategias de ABP. Sabiendo que la educación es un proceso lógico y secuencial que se encarga de la formación integral de los individuos (estudiantes), es más que evidente que la persona que está a cargo de dicho proceso, es decir el docente, debe realizar su función de guía de la manera más acertada posible, lo cual conlleve a propiciar un verdadero espacio de enseñanza aprendizaje, aprovechando los recursos existentes y acoplándose a las necesidades del contexto, así como técnicas y estrategias.

Esta premisa es corroborada según (Del Valle, 2009) el docente debe crear o buscar diferentes estrategias y teorías y satisfacer la necesidad de prepararse ante nuevas competencias. Hasta el momento todas las teorías del aprendizaje se han enfocado al aula presencial, así como las estrategias de enseñanza y evaluación. En el siglo XXI, surge la exigencia de desarrollar otras competencias y estilos en materia de e-learning, e-portafolio, habla del aprendizaje basado en el estudiante y colaborativo. Se debe tener presente que en el momento y entorno de estas teorías la tecnología no tenía el impacto de hoy día.

Como docente y tutor, se observa varios problemas en los estudiantes que vienen de diversas unidades educativas públicas hacia una particular específicamente al octavo año de EGB, como: es el bajo nivel de participación, observación, análisis, interpretación y exposición de trabajos, sus rendimientos se reflejan con notas menos de siete. Por lo tanto, los alumnos argumentan que se imparten las clases a partir de un modelo 
tradicional, donde el docente es el protagonista. Frente a esta problemática se pretende implementar alternativas metodológicas activas para innovar el aprendizaje y desarrollar la actitud investigativa científica en los estudiantes, garantizando una educación de calidad y calidez.

(Argentina, 2017) describe las estrategias de participación activa son esenciales durante el desarrollo de los contenidos en las diferentes disciplinas, permitiendo la integración de todos los estudiantes desde el inicio hasta el final de la clase. Cabe destacar que de lograrse a plenitud lo antes dicho el resultado sería un aprendizaje significativo y de calidad en cada estudiante. Es importante mencionar la demanda de los estudiantes de clases dinámicas y motivadoras, que despierten en los mismos, intereses y deseos de aprender de una manera afectiva y de confianza entre docente y estudiantes.

En base a la problemática presentada es importante elaborar el siguiente documento científico que permita mediante el reporte del rendimiento, entrevistas y fichas de observación medir el nivel de participación de los estudiantes conjuntamente con el ABP como técnica didáctica mejorar el nivel de participación y para adquirir nuevos conocimientos en la asignatura de Ciencias Naturales; considerando como una estrategia de innovación en la asignatura de Ciencias Naturales.

Según la Unesco las técnicas de recolección y análisis de datos como la observación, entrevista abierta y la triangulación, y bajo el alero del paradigma descriptivo permite obtener mejores resultados que mejoran sus habilidades de auto-aprendizaje, cada vez que recolecta información, estudia, organiza y presenta resultados (Paredes, 2014). En un trabajo elaborado por (Contreras, 2015) ha podido determinar que el ABP permite desarrollar competencias para resolver los problemas que pueden surgir a partir de los contenidos que se desarrollan sobre el medio natural. Así mismo, se explica que la enseñanza-aprendizaje de las Ciencias Naturales a través del ABP propicia la generación del nuevo conocimiento sobre la base de los conocimientos previos. Además, genera la curiosidad, el interés y la iniciativa por aprender contenidos desconocidos. Es una estrategia centrada en el estudiante como protagonista principal de su aprendizaje. 
Como se cita es importante aplicar este tipo de estrategia que está contribuyendo en la enseñanza de las Ciencias Naturales, por ello se plantea como objetivo general de este trabajo optimizar la participación de los educandos, a través de la aplicación del ABP como estrategia didáctica para la enseñanza en Ciencias Naturales. Por lo que el ABP se sustenta en la teoría constructivista, la idea central de este enfoque es que los estudiantes son quienes construyen activamente su conocimiento, dejan de ser los receptores de información para convertirse en el centro del aprendizaje (Gutiérrez, De la Puente, \& Piña, 2017).

Según la hipótesis planteada: ¿El ABP (Metodologías Basado en Problemas) como estrategia didáctica permitirá mejorar la enseñanza en la asignatura de Ciencias Naturales y los estudiantes mejoren significativamente? Se sustenta en las variables la independiente: implementación de la metodología ABP que Jofré (2015) manifiesta que al aplicar el ABP como estrategia metodológica en el aula, se ha observado que los estudiantes desarrollan habilidades como la comprensión lectora, capacidad de análisis, síntesis y relación de información, entre otras. Estas habilidades se potencian debido a que deben lograr identificar una problemática inmersa en la redacción del caso problema (Jofré \& Contreras, 2015).

Y en relación a la variable dependiente: enseñanza de Ciencias Naturales, favorece en los resultados debido a que con la aplicación de la metodología ABP se fomenta en mayor porcentaje el desarrollo de algunas competencias básicas comparándola con la metodología tradicional, así mismo, con los cuestionarios los estudiantes están adaptados a la metodología tradicional, pues por mucho tiempo han sido seres pasivos dentro del ámbito educativo.

Por lo que dedican poco tiempo a la elaboración de trabajos escolares o consultas de investigación, evidenciando que los estudiantes presentan resistencia inicial por la nueva metodología pues esta necesita más tiempo y dedicación por parte de ellos, se encuentran en una zona de confort, pero cuando se logra romper estas barreras los estudiantes empiezan a desarrollar diversas habilidades para la investigación, pueden 
asociar los saberes escolares a su contexto, se genera también mayor autonomía y participación en los grupos de trabajo (Cardona, Mora, \& Velásquez, 2017).

Por ello se presenta este trabajo muy importante, que se rige a fuentes bibliográficas importantes es en dónde (Chávez, González, \& Hidalgo, 2016) define a las estrategias ABP como una técnica didáctica centrada en el alumno, en ella el docente plantea un problema clínico inicial, complejo y retador para que sea resuelto basándose en el trabajo colaborativo dentro de grupos de trabajo pequeños, con el objeto de desencadenar el aprendizaje auto-dirigido.

Refuerza esta idea el Ministerio de Educación, Cultura y Deporte mencionando que este tipo de estrategia forma parte de un aprendizaje activo, conjuntamente con el aprendizaje basado en proyectos, en tareas, en problemas, y en retos; por lo que el conocimiento no es una posesión del docente que deba transmitir a los estudiantes sino más bien un proceso de trabajo entre docentes y estudiantes, considerando el papel del estudiante no solo como a escuchar sino a ser partícipe y el docente crear la situación de aprendizaje que permita que los estudiantes puedan desarrollar sus proyectos (Marti, Heydrich, Rojas, \& Hernández, 2015).

La implementación se realizará en los estudiantes del octavo año de EGB del Colegio de Bachillerato Particular Justiniano Crespo Verdugo del Cantón Cañar durante el lectivo 2019-2020, como una estrategia de innovación en la cuarta unidad, en la asignatura de Ciencias Naturales. Las experiencias de valoración de los estudiantes dentro del aula se siguen de manera continua y constante, con el grupo de control y el de intervención; mediante las actividades individuales, grupales, mesa redonda, discusiones, talleres, observación de campo, con amplia revisión bibliográfica.

Frente a este artículo se presentaron algunas limitaciones como ampliación de los aspectos analizados, poca conexión entre los docentes de diferentes áreas para poner en práctica el tema investigado, consideración de otros aspectos en el área investigada y la escasez de trabajos realizados sobre el tema de ABP como estrategias en las Ciencias Naturales; limitaciones que fueron asumidas y tratadas de la mejor manera. 
En base a ello es de considerar de como propuesta para aplicar esta estrategia es importante el manejo de las 5 modelos que describe una secuencia de enseñanza que se puede utilizar para programas completos, unidades específicas y lecciones individuales. Los planes de clase tienen como base el ciclo de aprendizaje constructivista que ayuda a los estudiantes a crear su propia comprensión (Bastida, 2018).

La educación científica tiene un papel importante que desempeñar en el desarrollo de ciudadanos informados y críticos en una sociedad en rápida evolución tecnológica, ya que se pretende lograr una comprensión del mundo en que vivimos. En esta propuesta de formación docente se ofrece un espacio curricular que revise los nuevos planteamientos y exigencias del medio social, cultural e histórico.

\section{MÉTODO}

La investigación se desarrolló desde una perspectiva cuantitativa, mediante el método hipotético deductivo y un tipo descriptiva correlacional transversal no experimental, mediante el cálculo de Chi-cuadrado de Pearson, con la finalidad de conocer la asociación de las variables de estudio (Carreño-Godoy, Erazo-Álvarez, Narváez-Zurita, \& Moreno, 2020).

Por ello este método permitió la recolección de datos en el cual se comparan las mediciones del comportamiento y aprovechamiento de los estudiantes del Colegio Particular Justiniano Crespo Verdugo, en las diferentes circunstancias educativas específicamente en la asignatura de las Ciencias Naturales (Boix, 2019).

La técnica empleada para la recolección de datos fue la encuesta, que es una técnica de recogida de datos mediante la aplicación de un cuestionario a una muestra de individuos. A través de las encuestas se pueden conocer las opiniones, las actitudes y los comportamientos de los estudiantes (Bautista, 2013). El instrumento utilizado es el formato de cuestionario de encuestas que está estructurado de la siguiente manera: Encabezado, a quienes está dirigida la encuesta, los objetivos, la forma de contestar y las preguntas que fueron de 10,3 para el objetivo general, 7 para los objetivos específicos, 
las preguntas están estructuradas de una forma que los encuestados puede contestar sin confusión por ellos las preguntas están entre abiertas y cerradas. A más de ello fue validado con Alfa de Cronbach, con el valor de 0,87.

El universo de estudio se realizó en el Colegio de Bachillerato Particular Dr. "Justiniano Crespo Verdugo" Cañar, su tratamiento muestral tomado de los estudiantes de octavo año, con una población de 40 alumnos. El total de la población fue de 40 estudiantes quienes permitieron que mediante las encuestas se obtengan datos muy valiosos, los mismos que fueron ingresados al sistema SPSS 22.0, para realizar el análisis, interpretación, la comparación de variables y confirmación de la hipótesis. Para de esta forma identificar el problema y partir desde la fundamentación para concluir el trabajo.

\section{RESULTADOS}

\section{Tabla 1}

Resultados utilización ABP

\begin{tabular}{|c|c|c|c|c|c|}
\hline Ítems & $\begin{array}{l}\text { Muy Alto } \\
5\end{array}$ & $\begin{array}{c}\text { Alto } \\
4\end{array}$ & $\begin{array}{c}\text { Medio } \\
3\end{array}$ & $\begin{array}{c}\text { Bajo } \\
2\end{array}$ & $\begin{array}{c}\text { Muy Bajo } \\
1\end{array}$ \\
\hline $\begin{array}{l}\text { Usted tiene problemas en la asignatura de } \\
\text { Ciencias Naturales }\end{array}$ & 7 & 0 & 13 & 8 & 12 \\
\hline $\begin{array}{l}\text { Que tanto aprende con el aula virtual cuando se } \\
\text { aplica el ABP }\end{array}$ & 10 & 24 & 5 & 0 & 1 \\
\hline $\begin{array}{l}\text { Como estudiante que nivel de conocimiento a } \\
\text { obtenido }\end{array}$ & 10 & 18 & 11 & 0 & 1 \\
\hline $\begin{array}{l}\text { Qué grado de responsabilidad asume ante el } \\
\text { aprendizaje. }\end{array}$ & 12 & 17 & 9 & 1 & 1 \\
\hline $\begin{array}{l}\text { Usted trabaja en grupos supliendo los posibles } \\
\text { problemas. }\end{array}$ & 4 & 6 & 18 & 5 & 4 \\
\hline $\begin{array}{l}\text { Usted tiene una actitud receptiva hacia el } \\
\text { intercambio de ideas con los compañeros. }\end{array}$ & 4 & 6 & 18 & 9 & 3 \\
\hline $\begin{array}{l}\text { Usted comparte información y aprende de los } \\
\text { demás. }\end{array}$ & 10 & 12 & 12 & 4 & 2 \\
\hline Usted es autónomo en el aprendizaje & 8 & 15 & 15 & 1 & 1 \\
\hline
\end{tabular}


(busca información, la contrasta, la comprende

y la aplica)

Sabe pedir ayuda y orientación cuando lo necesita.

Dispone usted de las herramientas necesarias para cumplir las actividades encomendadas por el docente.
12

15
$14 \quad 12$

14
6

4
1

\section{Fuente: Autoría propia}

Por lo que se evidencia en los siguientes resultados de forma general sobre las encuestas relacionados a los estudiantes; se puede conocer que existe un mayor porcentaje relacionado a un nivel medio de los estudiantes quienes si presentan problemas en la asignatura de Ciencias Naturales, así como un alto nivel de estudiantes que creen que con la aplicación de la estrategia de ABP puede aprender desde el aula virtual, mediante la plataforma EVEA de la Universidad Católica de Cuenca; conociendo que existe un nivel de conocimiento medio como estudiante, de la misma forma manteniendo una responsabilidad de aprendizaje alto, y en un nivel medio el trabajar en grupos cumpliendo con los posibles problemas.

Se evidencia problemas de Ciencias Naturales y la importancia del ABP como estrategia de enseñanza aprendizaje, por el nivel medio que hay en cuanto al estudiante cuando recepta e intercambia ideas con sus compañeros; y entre el nivel alto y medio cuando comparte información y aprende de los demás; buscando un aprendizaje autónomo conjuntamente con la disposición de herramientas para cumplir las actividades.

En relación a ello se presenta de forma general resultados en dónde se evidencia un nivel medio y bajo en sobre los problemas que presentan los estudiantes en Ciencias Naturales $y$ un medio $y$ alto sobre el uso de estrategias de ABP para mejorar dicho problema presentado por los estudiantes.

Considerando a la hipótesis ¿El ABP (Aprendizaje Basado en Problemas) como estrategia permitirá mejorar la enseñanza en las Ciencias Naturales? Considerando las 
dos preguntas que se plantean en el cuestionario ¿usted tiene problemas en la asignatura de Ciencias Naturales? y ¿Que tanto aprende con el aula virtual cuando se aplica el ABP? Hipótesis Nula $(\mathrm{H} 0)$

Los problemas en la asignatura de Ciencias Naturales de los estudiantes no están relacionados con la falta de estrategias para mejorar su aprendizaje.

Hipótesis Alternativa $(\mathrm{H} 1)$

Los problemas en la asignatura de Ciencias Naturales de los estudiantes están relacionados con la falta de estrategias para mejorar su aprendizaje.

Con el nivel de significancia del 5\% con grados de libertad de 9 según la tabla de valor de Chi-cuadrado tenemos un valor límite de 16,91, y según el análisis realizado nosotros tenemos un valor de Chi-cuadrado de 9,57. Donde debemos concluir indicando que dentro de un rango determinado se encuentra el resultado denominado zona de aceptación, por lo que la hipótesis que queríamos demostrar son aceptadas, es decir que los problemas en la asignatura de Ciencias Naturales de los estudiantes están relacionados con la falta de estrategias como el uso del ABP.

\section{Tabla 2}

Análisis de relación entre problemas en la asignatura de Ciencias Naturales y la falta de estrategias para mejorar su aprendizaje

\begin{tabular}{lccc} 
& Valor & gl & $\begin{array}{c}\text { Sig. asintótica } \\
\text { (bilateral) }\end{array}$ \\
\hline Chi-cuadrado de Pearson & & 9 & 0,038 \\
Razón de verosimilitudes & $9,570^{a}$ & 9 & 0,184 \\
Asociación lineal por lineal & 12,561 & 1 & 0,671 \\
N de casos válidos &, 181 & &
\end{tabular}

a. 14 casillas $(87,5 \%)$ tienen una frecuencia esperada inferior a 5 . La frecuencia mínima esperada es ,18.

Fuente: Elaboración propia

Sobre los resultados que se han elaborado se ha planteado como técnica de recolección de datos la encuestas; en dónde se la aplicó a un total de 40 estudiantes del Colegio 
Particular "Justiniano Crespo Verdugo" anexo a la Universidad Católica De Cuenca Sede Cañar; se ha evaluado puntos como si los estudiantes tienen problemas en la asignatura de Ciencias Naturales, en dónde el mayor puntaje es 5 como muy alto y 1 como muy bajo, se ha demostrado que existe un nivel medio y muy bajo en un total de 25 estudiantes; siendo recurrente con el trabajo de (Carranza, 2016), quien concibe que no necesariamente el desarrollo del alumno juega un papel limitante en el aprendizaje, sino el abordaje de la disciplina con un tema poco natural a los estudiantes a ese nivel.

Se recomienda cambiar el orden de los contenidos, empezando con el tema de fuerza y energía, haciendo un uso limitado de herramientas o estrategias, al menos en las etapas iniciales del aprendizaje. Reducir el número de contenidos del programa y poner en práctica una serie de estrategias didácticas que aumenten el carácter significativo del aprendizaje para el alumno, entre las cuales se cuentan resolver problemas de la vida cotidiana con el uso del método científico, por lo que se evidenció un total de $65 \%$ de estudiantes con problemas en esta asignatura (Carranza, 2016).

De esta manera se evidencian problemas en la enseñanza y aprendizaje de esta materia, muchos docentes aplican diferentes técnicas y estrategias para mejorar los resultados en pocas ocasiones la ABP, es así que en este estudio un total de 34 estudiantes consideran que este tipo de estrategia mediante el aula virtual permitiría aprender de mejor manera. Se ha evaluado el nivel de conocimiento de los estudiantes determinando que existe el mismo que es alto y medio, asumiendo un nivel alto en la responsabilidad sobre su aprendizaje, tratando de suplir los problemas mediante el trabajo grupal. Este resultado se refuerza con un estudio elaborado por (Cardona, Mora, \& Velásquez, 2017), quien propuso la estrategia didáctica del aprendizaje basado en problemas (ABP), para fortalecer las competencias como el uso comprensivo del conocimiento científico, la explicación de fenómenos y la indagación.

Cuando el estudiante trabaja con sus compañeros un nivel medio tienen una actitud receptiva hacia el intercambio de ideas con sus compañeros, así como la compartición de ideas y el aprender de los demás, en un nivel medio el ser un estudiante autónomo es decir busca información, la contrasta, la comprende y la aplica en la resolución de 
problemas. Los resultados en relación a que si el estudiante sabe pedir ayuda y orientación cuando lo necesita un gran nivel considerado como alto del total de estudiantes lo hace y disponen de herramientas necesarias para cumplir con estas actividades encomendadas.

\section{PROPUESTA}

A continuación, se aborda una propuesta alternativa para el uso de del ABP basado en las $5 \mathrm{E}$ (enganchar, explorar, explicar, elaborar y evaluar), en la asignatura de Ciencias naturales. Los vértices del pentágono propuestos no requiere grandes adaptaciones curriculares, su implantación es muy fácil que contribuye en un potencial óptimo de los estudiantes, durante el proceso de aprendizaje.

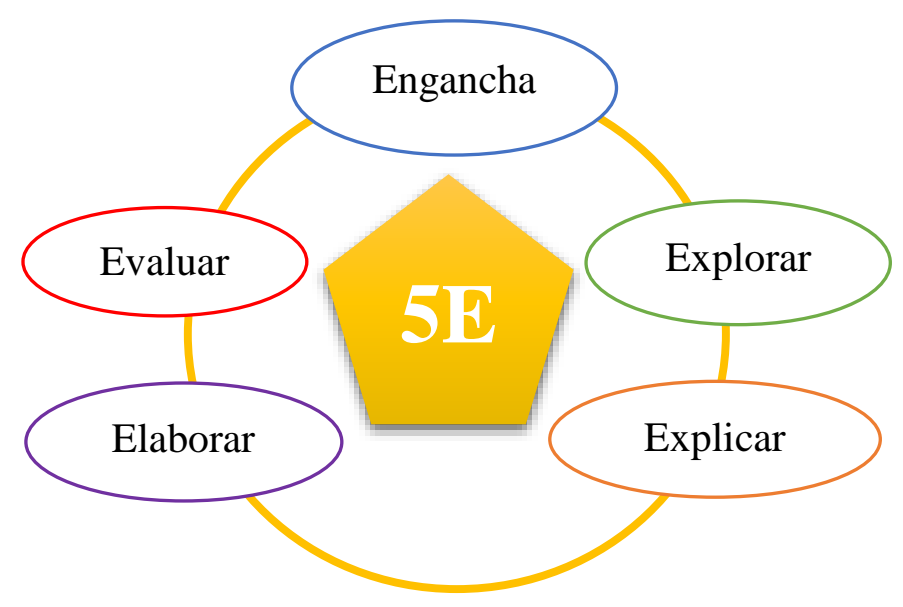

Figura 1. Las $5 \mathrm{E}$

Se propone que el docente fomente una estrategia para estimular y motivar a los estudiantes en forma individual y colectiva, los primeros cinco minutos de la clase es propicio para que el docente transmita, una actitud entusiasta que les permita superar la posible frustración al momento de realizar un experimento; incitar a que recobren confianza en sí mismos para asumir conscientemente que los errores cometidos en la 
experimentación sirva para lograr una solución que deben ser vistos como oportunidades de aprendizaje.

Cabe destacar, que la implementación de la propuesta ha sido posible gracias a que la institución educativa que cuenta con proyectores multimedia e internet dentro de las aulas de clases y también está dotada con laboratorios de Ciencias Naturales. Uno de los componentes más importantes es que el docente estructura el plan de unidad didáctica (PUD), aplicando cíclicamente el modelo de las cinco 5E bajo una secuencia diseñada específicamente de la siguiente forma:

Enganchar, consiste en dialogar con los estudiantes sobre la valiosa oportunidad que significa participar durante los eventos educativos, tales como: el Concurso Interno coordinado por el área de Ciencias naturales, casa abierta, el día del agua, del medio ambiente etc.

Al iniciar la clase los estudiantes son motivados con la presentación de un video de 3 a 5 minutos, seguidamente emiten sus opiniones y luego se les pide que asuman un rol en el que imaginariamente ellos son un grupo de expertos en la ciencia natural y que el equipo de científicos del Ecuador necesita su talento profesional para la elaboración de proyectos ambientales, que permitan resolver problemas sociales.

El proceso debe ser guiado con la finalidad de despertar interés en los estudiantes sobre el contenido específico de la clase sin limitar que surjan inquietudes sobre otros contenidos. Previo a esta fase, el docente explica que ingresen al aula virtual para que cada estudiante pueda estar identificado en la plataforma EVEA.

Para tal finalidad, se debe socializar el programa de estudio y la familiarización con la plataforma virtual y hacer énfasis en la importancia del acompañamiento para la efectividad del proceso educativo.

Explorar, organizados en grupos de cuatro integrantes, el docente entrega un material a los expertos para que se paseen por un proceso de indagación, en el que también realizan investigación al consultar videos sobre el tema y se le asignan actividades de refuerzo para realizar en casa desde la plataforma virtual. 
Explicar, los equipos de expertos explican con sus propias palabras las ideas acerca de los contenidos estudiados, hacen representaciones y comparten sus ideas con los integrantes del grupo de trabajo. Durante el proceso son guiados a través de preguntas que formula el docente con la intencionalidad de propiciar la construcción de ideas más completas y profundas, en un ambiente que los motiva a explicar sus ideas y entendimientos.

Elaborar, los expertos, es decir, los estudiantes trabajan de manera colaborativa para realizar actividades experimentales, utilizando en varias ocasiones material reciclado.

De este modo les permite a los equipos de expertos ayudar a utilizar sus saberes y habilidades previas, a encontrar respuestas alternativas a sus inquietudes y a profundizar sus conocimientos sobre los temas de estudio.

En esta fase del ciclo de aprendizaje los equipos de expertos se involucran en la solución de nuevas situaciones elaborando dípticos, trípticos de proyectos ambientales con creatividad combinando los conocimientos de todas las asignaturas.

Evaluar, los equipos de expertos exponen sus proyectos en esta atapa final y reciben retroalimentación para conocer la exactitud de sus explicaciones y habilidades. Se realiza la evaluación utilizando rúbricas que toman en consideración los siguientes criterios: la pertinencia, el impacto social, la calidad del empleo de herramientas, creatividad, estética y la calidad del trabajo en equipo.

En este momento el docente motiva a los estudiantes a través del reconocimiento de sus esfuerzos, se valoran las habilidades que han adquirido, se evalúa la comprensión e incluso se retroalimenta el proceso con consideraciones que mejoren la capacidad de comunicar las soluciones.

Las actividades individuales son asignadas en la plataforma, donde los estudiantes deben alcanzar la máxima calificación dado que el sistema está configurado para que puedan presentar las lecciones y las pruebas, con varios intentos hasta lograr el $100 \%$.

El proceso de evaluación es continuo y el docente debe observar las actuaciones de los estudiantes en los grupos de trabajo durante todas las fases de las $5 \mathrm{E}$. Evaluar es ir mucho más allá y es un proceso complejo por el cual el docente debe propiciar los medios 
y orientar a los estudiantes al logro, y, esto debe hacer posible que las calificaciones mejoren.

El docente debe tener presente que las calificaciones son indicadores por el cual ha sido capaz de guiar a los estudiantes sobre un efectivo proceso de aprendizaje. Bajo ninguna circunstancia el docente debe asumir una actitud castigadora en caso de que un estudiante cometa un error en el proceso.

Importante en caso de una adaptación curricular por necesidades educativas especiales (NEE), en el PUD se deben considerar las recomendaciones del departamento de consejería estudiantil (DECE), esto permitirá que el docente disponga de más tiempo en el aula para brindar mayor atención a los estudiantes que lo ameritan, es decir, es una combinación de recursos metodológicos y herramientas pedagógicas que hacen más inclusivo al proceso educativo.

\section{CONCLUSIONES}

Según los resultados obtenidos en la investigación se concluye manifestando que, en la asignatura de ciencias naturales, de octavo año de EGB del Colegio de Bachillerato Particular "Justiniano Crespo Verdugo", se fortalecieron mediante la estrategia didáctica del aprendizaje basado en problemas (ABP), por cuanto permitió desarrollar en los estudiantes el pensamiento científico, crítico y reflexivo.

La estrategia didáctica del aprendizaje basado en problemas (ABP), impactó en los estudiantes a quienes les permitió el desarrollo de habilidades comunicativas, de trabajo en equipo e interpersonales; de competencias básicas y científicas; de una actitud positiva frente al aprendizaje y adicionalmente.

El impacto de la investigación en el Colegio de Bachillerato Particular Justiniano Crespo Verdugo, es que se logró incluir con el apoyo de los docentes y directivos docentes, en el plan de asignatura de ciencias naturales el aprendizaje basado en problemas, como una estrategia pedagógica para fortalecer las competencias científicas.

La aplicación y análisis de la prueba de diagnóstico permitió conocer el nivel de las competencias científicas, evidenciando un alto porcentaje de estudiantes, con problemas 
en Ciencias Naturales; y un nivel alto en hacer uso del aula virtual cuando se aplica el ABP en Ciencias Naturales.

La resolución de los problemas por parte de los estudiantes, permite cambiar estrategias donde el alumno pueda asociar sus saberes con su diario vivir, pasando de ser acumuladores de información a saber comprender; y poder discernir que el hijo sabio alegra al padre, pero el hijo necio es tristeza de su madre Proverbios 10:1. Por lo tanto dejo abierto para que se realice una investigación a futuro.

\section{FINANCIAMIENTO}

No monetario.

\section{AGRADECIMIENTO}

A todo el talento humano del Colegio Particular Justiniano Crespo Verdugo por apoyar el desarrollo de la investigación.

\section{REFERENCIAS CONSULTADAS}

Argentina, G. (2017). Estrategias que permitan mejorar la participación activa durante el proceso de aprendizaje en estudiantes de Formación Docente de la Escuela Normal José Martí de Matagalpa [Strategies to improve active participation during the learning process in Teacher]. Estelí. Obtenido de https://repositorio.unan.edu.ni/7588/

Bastida, D. (2018). Adaptación del modelo 5E con el uso de herramientas digitales para la educación: propuesta para el docente de ciencias [Adaptation of the $5 \mathrm{E}$ model with the use of digital tools for education: proposal for the science teacher]. Revista Científica. doi:http://dx.doi.org/10.14483/23448350.13520

Bautista, L. (2013). Técnicas de recolección de datos [Data collection techniques]. Madrid: Print Services.

Boix, J. (2019). Introducción a la fonética: el método experimental [Introduction to phonetics: the experimental method]. México: Mercedez. Obtenido de Método experimental. 
Campos, O. (2013). La enseñanza del emprendimiento a partir del aprendizaje basado en problemas $(A B P)$ en la educación media técnica [Teaching entrepreneurship from problem-based learning $(A B P)$ in technical secondary education]. Caquetá: Florencia. Obtenido de LA ENSEÑANZA DEL EMPRENDIMIENTO A PARTIR DEL APRENDIZAJE BASADO EN PROBLEMAS (ABP) EN LA EDUCACIÓN MEDIA TÉCNICA.

Cardona, M., Mora, R., \& Velásquez, L. (2017). ABP para fortalecer las competencias básicas en la institución educativa rural Santa Ana [ABP to strengthen basic skills in the rural educational institution Santa Ana]. Universidad Pontificia Bolivariana, Puerto Asís - Putumayo.

Carranza, C. (2016). Dificultades que enfrentan los estudiantes de $10^{\circ}$ año en el estudio de física. Alternativas para mejorar el aprendizaje [Difficulties 10th grade students face in studying physics. Alternatives to improve learning]. Ensayos Pedagógicos, 1,101-113. Obtenido de https://n9.cl/hga0w

Carreño-Godoy, M. J., Erazo-Álvarez, J. C., Narváez-Zurita, C. I., \& Moreno, V. P. (2020). La responsabilidad social en las empresas camaroneras [Social responsibility in shrimp companies]. Revista Arbitrada Interdisciplinaria Koinonía, 455-482. doi:http://dx.doi.org/10.35381/r.k.v5i10.702

Chávez, G., González, B., \& Hidalgo, C. (2016). Aprendizaje Basado en Problemas (ABP) a través del m-learning para el abordaje de casos clínicos. Una propuesta innovadora en educación médica [Problem Based Learning (ABP) through mlearning to approach clinical cases. An innovative proposal in medical ]. Innovación Educativa, 72(16), 1665-2673 . Obtenido de https://n9.cl/9bjh4

Contreras, M. (2015). "El Aprendizaje Basado en Problemas (ABP) para la enseñanza de las Ciencias Naturales en Quinto Año de Educación General Básica" ["Problem Based Learning $(A B P)$ for the teaching of Natural Sciences in the Fifth Year of Basic General Education"]. Universidad Nacional de Educación. Obtenido de https://n9.cl/i0b0

Gutiérrez, J., De la Puente, G., \& Piña, E. (2017). Aprendizaje Basado en Problemas un camino para aprender a aprender [Problem Based Learning a way to learn to learn]. México: Colegio de Ciencias y Humanidades.

Instituto Tecnológico y de Estudios Superiores de Monterrey. (3 de Febrero de 2016). $A B P$ como técnica Didáctica [Project-based learning]. Obtenido de https://n9.cl/082w 
Jofré, C., \& Contreras, F. (2015). Implementación de la Metodología ABP (Aprendizaje Basado en Problemas) en Estudiantes de Primer año de la Carrera de Educación Diferencial [Implementation of the ABP Methodology (Problem Based Learning) in First Year Students of the Differential Educatio]. Estudios Pedagógicos. doi:http://dx.doi.org/10.4067/S0718-07052013000100006

Marti, J., Heydrich, M., Rojas, M., \& Hernández, A. (2015). Aprendizaje basado en proyectos [Project-based learning]. EAFIT, 46(58), 11-21. Obtenido de https://n9.cl/fj3s

Paredes, C. (2014). Aprendizaje basado en problemas (ABP): Una estrategia de enseñanza de la educación ambiental, en estudiantes de un liceo municipal [Problem-based learning (ABP): A teaching strategy of environmental education, in students of a municipal high school]. Educare, 23-36. doi:http://dx.doi.org/10.15359/ree.20-1.6

Vélez-Loor, M., Vallejo-Valdivieso, P., \& Moya-Martínez, M. (2020). Recursos didácticos virtuales en proyectos de ciencias naturales en período de confinamiento por COVID-19. [Virtual teaching resources in natural science projects in confinement period by COVID-19]. EPISTEME KOINONIA, 3(5), 183-201. http://dx.doi.org/10.35381/e.k.v3i5.760 